\title{
Modeling of Quality Parameter Values for Improving Meshes
}

\author{
O. Egorova*, M. SAVChenko ${ }^{\dagger}$, I. HagiWARA ${ }^{\ddagger}$ and V. SAVChEnKo ${ }^{\S}$ \\ * Tokyo Institute of Technology, \\ 2-12-1, O-okayama, Meguro-ku, Tokyo, Japan \\ E-mail: egorova.o.aa@m.titech.ac.jp \\ $\dagger$ InterLocus. Inc. \\ 2-12-1, O-okayama, Meguro-ku, Tokyo, Japan \\ ¥ Tokyo Institute of Technology, \\ 2-12-1, O-okayama, Meguro-ku, Tokyo, Japan \\ $\S$ Hosei University, \\ 3-7-2 Kajino-cho, Koganei-shi, Tokyo, Japan
}

Received October 26, 2004

Revised January 11, 2007

\begin{abstract}
A novel quasi-statistical approach to improve the quality of triangular meshes is presented. The present method is based on modeling of an event of the mesh improvement. This event is modeled via modeling of a discrete random variable. The random variable is modeled in a tangent plane of each local domain of the mesh. One domain collects several elements with a common point. Values of random variable are calculated by modeling formula according to the initial sampling data of the projected elements with respect to all neighbors of the domain. Geometrical equivalent called potential form is constructed for each element of the domain with a mesh quality parameter value equal to the modeled numerical value. Such potential forms create potential centers of the domain. Averaging the coordinates of potential centers of the domain gives a new central point position. After geometrical realization over the entire mesh, the shapes of triangular elements are changed according to the normal distribution. It is shown experimentally that the mean of the final mesh is better than the initial one in most cases, so the event of the mesh improvement is likely occurred. Moreover, projection onto a local tangent plane included in the algorithm allows preservation of the model volume enclosed by the surface mesh. The implementation results are presented to demonstrate the functionality of the method. Our approach can provide a flexible tool for the development of mesh improvement algorithms, creating better-input parameters for the triangular meshes and other kinds of meshes intended to be applied in finite element analysis or computer graphics.
\end{abstract}

Key words: random variable modeling, randomness, triangulation, mesh quality parameter, mesh improvement

\section{Introduction}

The purpose of the present section is to give a survey of mesh improvement algorithms and show the score of our method. The mesh improvement is almost an obligatory step for obtaining a valid finite element mesh. In spite of a flurry of activities in the field of the mesh modification, the mesh improvement remains a difficult and computationally expensive problem.

There are many improvement techniques for the optimal point placement (see [1] and references therein). Most of them are based on the idea of the local optimization and require the improvement of such mesh quality parameters as aspect ratio (AR), area, etc. Global mesh optimization has been also studied recently [2]. In spite of the well-known fact that a local enhancement does not often provide reliable results, local methods are preferable in many applications with a rather large set of polygons, 
because the computational time is much less than that of global-based techniques. In fact, these methods can be called deterministic. Recently, an implementation of the statistical approach to the mesh quality parameter modeling was discussed in [3]. The aspect of the suggested idea is adjusted in the present paper. For practical applications, it is necessary that the results of a mesh improvement allow the construction of the topologically correct meshes. However, it is very difficult or even impossible to generate a mesh with elements corresponding exactly to the ideal mesh quality parameter values (in the case of triangular mesh equal to unity values) if topology is strictly preserved. The goal of the presented research is to evoke an event of the shape improvement of the triangles with respect to the topological structure of the mesh by producing the elements with the normal distribution of selected mesh quality parameter values. Gaussian distribution function is taken in the modeling formula to achieve the global consistency of the event. Local modeling and the realization of few numbers temper the randomness that may occur. Proposed method can be used in a pre-processing stage for subsequent studies (finite element method (FEM [4]), computer graphics (CG), etc.) based on triangle meshes by providing the better-input parameters for these processes.

In order to point out some properties of the suggested method and to show that its concept is totally different from existing ones, let us here briefly overview the methods of the mesh improvement related to computer-aided design (CAD) and computer-aided engineering (CAE) applications.

There are two main ways of improving the mesh quality: (1) modification of the mesh topology by inserting or deleting nodes, or by local reconnection (though it is sometimes necessary to minimize the changes in the topology of a surface) and (2) smoothing technique. In [5], a smoothing technique is defined as a means of correcting poorly shaped elements; that is, smoothing is an operation for producing elements that closely resemble theoretical elements.

Laplacian smoothing $[6,7]$ and its variations are the earliest of mesh improvement methods. In this technique, nodes are moved into the areas of poorly conditioned elements of the mesh. The Laplacian algorithm locates an offending node and moves it to the centroid of the surrounding nodes for improving the shape of the elements. On some non-convex domains, nodes can be pulled outside the boundary. An angle smoother in 2D, which tends to mount torsion springs between nodes and to minimize the system energy, was proposed in [8]. In optimization-based methods so-called the cost function is minimized, instead of moving each node in the basis of some geometric characteristic as done in Laplacian and angle-based smoothing. There are several kinds of the cost functions: minimum (maximum) angle [9, 10], aspect ratio [11] and distortion metrics [12, 13, 14]. While optimization-based methods are very effective to avoid the invalid elements, the computational time is much longer than that of Laplacian and angle-based techniques. Therefore in $[9,12]$ the authors advocate that a combined Laplacian/optimization-based approach should be applied. In the physically based methods, it is assumed that nodes are moved under the influence of some forces so that the shape of the incident elements is improved. In $[15,16]$, the authors consider a mesh as a system of springs that exert 
repulsive or attractive forces. In the methods [17, 18], nodes are moved to attain equilibrium at centers of bubbles. Some physically based methods can be classified as the optimization-based techniques. For instance, in [19] the forces at each node are considered. The simulation of these forces and the minimum energy configuration are defined by using an optimization procedure.

In the recent years, CG community has paid more attention to the mesh smoothing based on a signal processing approach, pioneered by Taubin in 1995 [20]. Rational filters offer an approach to the mesh smoothing [21], where a Laplacian operator and a curvature operator are used to remove undesirable noise. Subdivision schemes are an alternative approach to the problem. Nevertheless, subdivision schemes [22] are able to deal with the arbitrary topology but not with the arbitrary connectivity, as mentioned by Kobbelt et al. in [23].

Despite the many practical applications of triangle meshes in CG, there are applications, which require well-shaped triangulations, for example a texture mapping technique. In FEM applications not only accuracy of calculations but also the speed may be sacrificed. Still well-shaped triangulations may be useful as mentioned in [24] "forty-odd years after the invention of the finite element method, our understanding of the relationship between mesh geometry, numerical accuracy, and stiffness matrix conditioning remains incomplete, even in the simplest cases." If a mesh is created for FEM applications, it is very important to control the mesh gradation smoothness. Shape elements have a strong influence on a discretization error. When the mesh gradations are not smooth, the discretization error of the neighboring elements changes markedly. From the physical point of view, this means that the relative stiffness of neighboring elements also changes significantly, which may cause problems in many tasks. For instance, all transient simulations (metal forming problems like forging, extrusion, rolling, etc., where the mesh of the workpiece evolves to represent the material flow) and heat transfer processes are concerned with tracking phenomenon that propagates through the domain. If stiffness changes are too large, propagation information can be made to reflect back incorrectly in the opposite direction, just as would occur when a stress wave hits the interface of materials of different stiffness [25]. One of the main properties of our quasi-statistical approach is that a uniform (homogeneous) mesh in the sense of the distribution of the element shapes is received that is more amenable to some numerical calculations. In general, our method allows the reduction in the number of very low quality elements avoiding overly good elements. It is achieved by means of a mesh uniformity and improvement of the average AR of a mesh if topology does not restrict. The method is designed to improve the geometrical shape of the mesh faces without distorting the discrete surface too much. In a special case when the neighbour elements of the mesh are still very bad, which can be recognized after one or two iterations of our algorithm, we use topological modification. Our method can be interpreted as a kind of the smoothing technique using modeling of the mesh quality parameters.

The use of the quasi-statistical modeling for the triangular mesh improvement is discussed in this paper. The rest of the paper is organized as follows. The explanation of the concept of the present approach is given in Section 2. The geometrical 
realization of quasi-statistical modeling is introduced in Section 3. Section 4 presents some examples and discussion on the properties of the method. Section 5 contains concluding remarks.

\section{Concept of the present approach}

Suppose a triangular mesh generated by some existing algorithm is given. Assume that all points are correctly connected in a topological structure depending on the initially given coordinates of the points. Such topology defines geometrical properties of the generated mesh. If a parameter of mesh quality is chosen, for example, the area, the minimum (maximum) angle, aspect ratio, or others, then a real number (a value of this parameter) can be put in accordance with each element of the mesh. These numbers can be regarded as random and one can perform a sampling analysis to check the mean value, the deviation and the distribution histogram. The normal distribution is the most widely used distribution to describe random variables. This is a reason for the selection of Gaussian distribution for our modeling of the mesh quality parameter values. We can put the aspect of the normal distribution appearance into the basis of the suggested approach and model the mesh improvement process. Our target can be formulated in terms of a random event as improving a mesh so that the mean value of the mesh quality parameter values becomes better. We have only two possibilities - if desired event occurs, then event indicator equals 1 and if it does not occur, then it is 0 .

It is a commonly used way in statistics to model an event through the modeling of a random variable. Therefore, we are planning to model a random variable with Gaussian distribution with respect to the initially given topology and the geometry of the mesh in order to cause a desirable event of the mesh improvement. The solution of the constructed equation (4) introduced below is based on $n$ determined numbers of the initial mesh quality parameter values. It gives us $n$ values of a random variable, which is being modeled. This random variable $\xi$ has Gaussian function laid in the equation as its distribution function. The general classification of the transformations for a random variable modeling was suggested in [26]. We omit here the precise description of the derivation of the suggested formula for the modeling step. Values of $\xi$ are calculated for each element from a local domain, which we call a star, collecting all elements with a common node. A geometrical operation in a tangent plane of a local domain is then provided for the connection between the geometry and $n$ received values of modeling random variable. Basic scheme of this procedure is demonstrated in Fig. 1. The left drawing corresponds to the initial probability density function $f(x)$ of the mesh elements of one star and the right drawing corresponds to the desired probability density function $g(y)$. Distribution functions $F(x)$ and $G(y)$, which correspond to density functions $f(x)$ and $g(y)$, have to satisfy the following parity $F\left(a_{i}\right)=G\left(a_{i}^{*}\right)$ for any initial value $a_{i}$ of the mesh quality parameter in order to model a new value $a_{i}^{*}$. Notice, that there are some cases when two or more different values from the initial distribution can be modeled into one new value $a_{i}^{*}$ of the random variable with the normal 


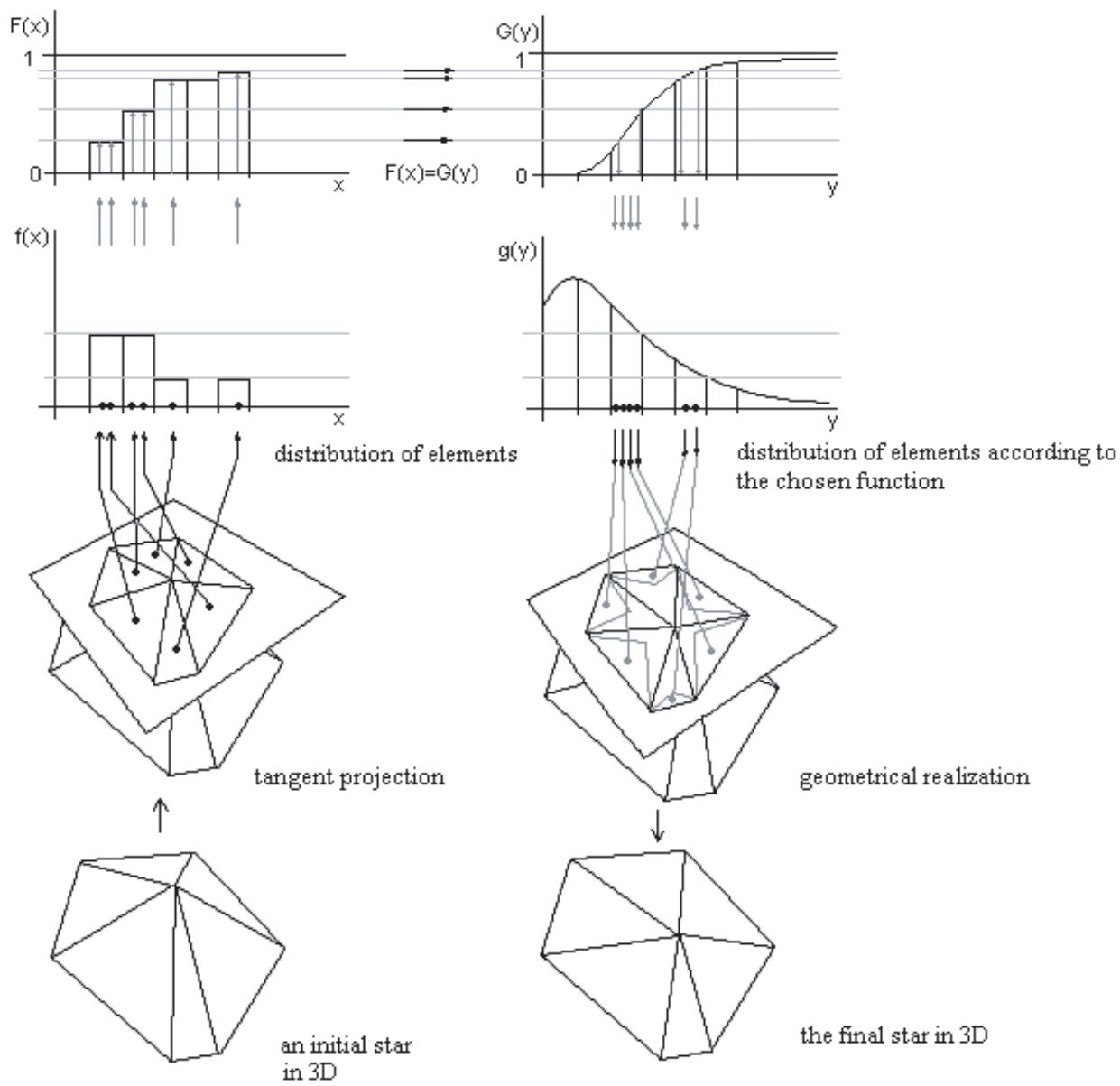

Fig. 1. Illustration of the algorithm implementation for one star.

distribution. Actually, it happens because we solve a discrete case of the equation, i.e. $F_{j}\left(a_{i}\right)=G_{h}\left(a_{i}^{*}\right)$. Thus, several different values $a_{i}$ and $a_{l}$ may come into one subinterval. Another important point here is that the geometrical realization of the modeled value $a_{i}^{*}$ changes the modeled value itself. This change after the geometrical realization is permitted because the modeled value $a_{i}^{*}$ is considered as a kind of statistical weight. After the entire mesh is treated, all the stars are considered and the geometrical realization is completed, more elements will have the new mesh quality parameter values near the peak of the normal distribution.

The AR is a main criterion chosen for further investigation of the algorithm. The AR value of each element of a current star is calculated in the tangent plane of this star, where $3 \mathrm{D}$ coordinates of the local boundary vertices are projected onto the plane (see [27]), and defined as a length of the longest edge divided by the length of the shortest edge of a triangle element. Such definition of the AR determines a 
range of values from 1 to the maximum value, i.e. probabilistic space $X=\left[1, a_{\max }\right]$. One can apply the probabilistic analysis over intervals $\left(n_{j}, n_{j+1}\right]$ (for some integer number $k$, where $\left.n_{0}=1, \ldots, n_{k}=a_{\max }\right)$, for the $\mathrm{AR}$ values equal to $a_{1}, \ldots, a_{n}$ for a current star. Statistical parameters such as the deviation $D$ from the average value $M$ can be found as follows:

$$
M=\frac{\sum_{i=1}^{n} a_{i}}{n}, \quad D=\frac{\sum_{i=1}^{n}\left(a_{i}-M\right)^{2}}{n} .
$$

The histogram values $f_{j}$ are as:

$$
f_{j}=\left|\left\{i: n_{j} \leq n_{j+1}\right\}\right| .
$$

Probability values $F_{j}$ for the AR values $a_{i}$ lying inside the interval $\left(n_{0}, n_{j+1}\right]$ are calculated by formula (3).

$$
F_{j}=\frac{\left|\left\{i: a_{i} \leq n_{j+1}\right\}\right|}{n+1}
$$

Empirical distribution number $F_{j}$ calculated by formula $(3)$ is assigned to all values $a_{i}: a_{i} \mapsto F_{j(i)}=F_{j}$. Finally, with the deviation $D$ from the average $M$ and probabilities $F_{j}$, one can produce new values $a_{1}^{*}, \ldots, a_{n}^{*}$ of the mesh quality parameter modeled by formula:

$$
a_{i}^{*}=\sqrt{-2 D \ln \left(1-F_{j}\right)}+1 .
$$

This modeling formula is applied to the elements of each operating star. Geometrical equivalent of the new values derived from this formula is constructed and determined in the tangent plane as shown in the bottom parts of Fig. 1. We call this geometrical equivalent a potential form or a geometrical realization. The way of a potential form construction will be explained in the next section. Ideologically, the left-hand part of Fig. 1 corresponds to the sampling data defined using formulas (1)-(3) while its right-hand part concerns the modeling of the new mesh quality parameter values for the neighbouring elements of each star by formula (4). Now imagine that this procedure is applied to each star. When all the points are investigated, we may check the final quality of the mesh by formulas (1)-(3) taking all $N$ mesh elements into consideration. Finally, forming a histogram for these $N$ elements, we can identify, whether the desired event occurs or not.

\section{Geometrical equivalent of modeled values}

As we already mentioned, the construction of the potential forms in our quasistatistical approach is implemented in a tangent plane of a local domain (star). The geometrical realization introduced here conforms to the corresponding projections of the mesh elements. The new mesh quality parameter values are modeled by formula (4) for such projections of the operating star. These values are judged as 
quasi-statistical weights of the initial elements with respect to a star. Improving a mesh with predefined potential mesh quality parameter values $a_{1}^{*}, \ldots, a_{n}^{*}$, we need to generate a topologically correct mesh in a star according to these values. To achieve this goal, we construct the potential forms for each triangle of a star. The potential forms define potential centers of a star. The new position of the central point of the star is calculated by averaging the coordinates of all potential centers of the star. Fig. 2 illustrates the geometrical realization process. We call such proceeding an operation on a star.

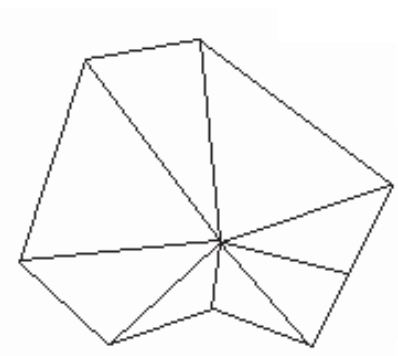

(a)

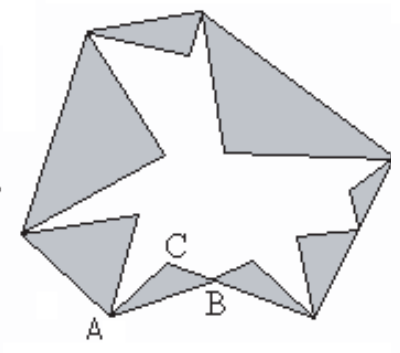

(b)

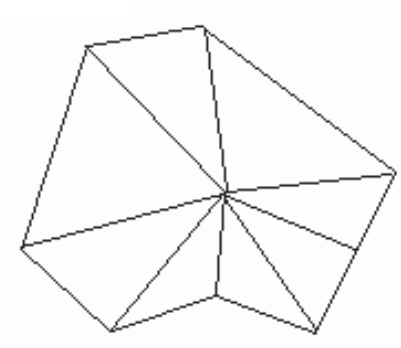

(c)

Fig. 2. Geometrical realization of modeling values: (a) an initial star; (b) potential forms (grey), C is a potential center; (c) the improved star.

Below, we explain the construction of a potential form for a triangular mesh element of a star on its fixed boundary edge. This potential form is a geometrical equivalent of a new mesh quality parameter value derived from probabilistic point of view.

Consider a star in a tangent plane with $n$ triangular elements as shown in Fig. 2 (a). Suppose that the boundary edges are fixed and the only one inner point of the star can freely slide within this plane. For each element with the fixed boundary edge $\mathrm{AB}$, we find a new position of the inner point $\mathrm{C}$ (potential center) according to value $a_{i}^{*}$ calculated by formula (4), see Fig. 2 (b). Such a position provides a potential shape $\mathrm{ABC}$ of this triangular element, which is an ideal one from probabilistic point of view with respect to the shapes of the current element's neighbors. Actually, there are various shapes of a triangular element corresponding to given $\mathrm{AR}$ value $a_{i}^{*}$ even with a fixed edge of the element. From given values $a_{i}^{*}$ and fixed side $\mathrm{AB}$, we determine a minimum side of the potential triangle computing $\mathrm{AB} / a_{i}^{*}$. It means we make an assumption that $\mathrm{AB}$ is a length of a maximum side of the potential element. This assumption is permitted by modeling step with Gaussian distribution, which already includes randomness that may occur at this stage as well as at the stage of the projection. Then draw a circle with radius $r=\mathrm{AB} / a_{i}^{*}$ and the center $\mathrm{B}$ forming many new triangles $\mathrm{ABC}$ (Fig. 3 (a)). Again, we need to select only one among them. We maximize the minimum angle $\alpha$ adjacent to $\mathrm{AB}$ in order to find one point $\mathrm{C}$ as a potential center. Fig. 3 (a) demonstrates that the angle $\alpha$ is maximal when the line $\mathrm{AC}$ is tangent 


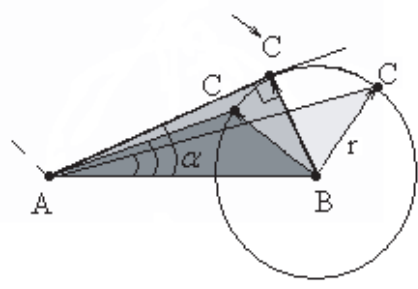

(a)

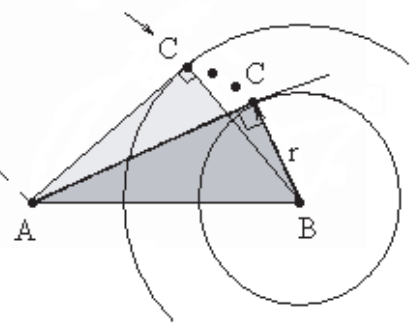

(b)

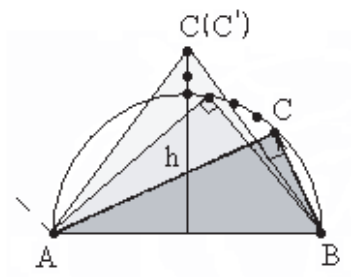

(c)

Fig. 3. Construction of the potential center $\mathrm{C}$ for one element of the star: (a) potential form for one modeled value (maximization of the minimum angle); (b) varying the radius $\mathrm{r}$ when the modeled value ranges; (c) the final locus of the points $\mathrm{C}$.

to the circle. Thus, a potential form is constructed for one mesh element.

Although we do not know what value of $a_{i}^{*}$ comes out during modeling, we need to understand all geometrical solutions that will be necessary to define a geometrical equivalent of the modeled value $a_{i}^{*}$. Thus allow the modeled value $a_{i}^{*}$ ranges over the probabilistic space $X$. As the radius $r$ changes, we receive a set of triangles based on $\mathrm{AB}$ with opposite angles $\mathrm{C}$ with 90 degree, i.e. the case when AC is tangent to the circle, see Fig. 3 (b). So, the opposite points of such triangles form the semicircle based on the diameter AB (Fig. 3 (c)). For example, the point $\mathrm{C}$ corresponding to the intersection of the semicircle $\mathrm{AB}$ and the height $h$, is appropriate to value $a_{i}^{*}=\sqrt{2}$, where $h=\mathrm{AB} / 2$. We conclude that semicircle AB corresponds to the range of the modeled value $a_{i}^{*}$ from $\sqrt{2}$ to $a_{\max }$, where $a_{\max }=\infty$, if $\mathrm{C}=\mathrm{B}$ or $\mathrm{C}=\mathrm{A}$. Notice also that the point $\mathrm{C}=\mathrm{C}^{\prime}$ corresponds to the ideal case of equilateral triangle, where $a_{i}^{*}=1$ and the height $h=\mathrm{AB} \sqrt{3} / 2$. So, the rest of the range of the modeled value $a_{i}^{*}$, i.e. from 1 to $\sqrt{2}$, is defined by the upper segment of the height $h$. Summarily, Fig. 3 (c) depicts the locus of the points C, when the length $\mathrm{AB}$ of the potential element $\mathrm{ABC}$ is fixed, but corresponding modeled number $a_{i}^{*}$ is varied. In real situation we select only one position of the point $\mathrm{C}$ for the given $\mathrm{AB}$ with regard to one modeled value $a_{i}^{*}$, which is uniquely defined by formula (4) for this mesh element of the star. Finally, constructing potential centers $\mathrm{C}$ for all neighboring elements of the star and averaging the coordinates of these points we determine the new coordinates of the central point of the star (Fig. 2 (c)) and a new point placement is completed.

Notice that each element will be considered three times in different stars. The reason is that a mesh element has three nodes in connectivity. This provides uniform change from star to star. Finally, the smoothness of each pair of neighbors of the mesh is guaranteed by produced formula for modeling of random variable with Gaussian distribution, which is implemented in each star. Usually other methods consider each element with the same possibility of changing its shape quality, deriving many high quality elements at the expense of some others. Refusing such kind of situation, we assign to each element different possibilities and finally generate more 
homogeneous mass of the uniform-quality elements in opposite to well-known mesh improvement techniques. Formula (4) can be interpreted as a connection between desired mesh quality and real possibilities of the mesh geometry and topology.

Fig. 4 shows three variants of the histograms of values $f_{j}$ calculated for investigated mesh fragment: an initial histogram of the mesh, the histogram of the improved mesh after method application according to all $a_{i}^{*}=1$ and according to modeled values by formula (4). The length of the fragmentation of an interval corresponding to probabilistic space $X$ is the same over entire model. The number of subintervals is very large on the Fig. 4 (a), where initial distribution is shown, but it is about three times less on the Figs. 4 (b) and 4 (c) than on the Fig. 4 (a). Light grey color regards to improved mesh after one iteration and dark grey - after two iterations. From the histograms we see that the distribution of AR values in case (c) is closer to normal distribution than in case (b) and the curve intents to centre at the ideal value 1 . The average value $M$ is improved in both cases.

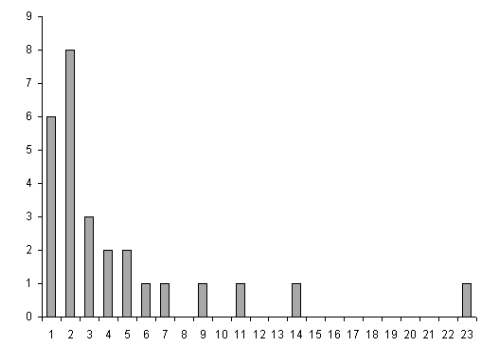

(a)

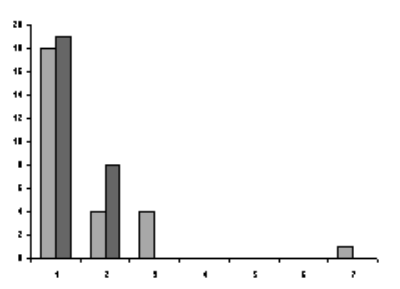

(b)

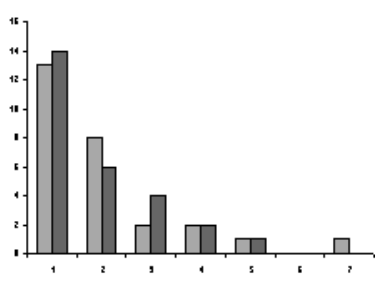

(c)

Fig. 4. Frequency histograms $((x)$ axis - the number of the intervals of the AR value range $X=[1.0, \max ] ;(y)$ axis - the number of the mesh elements): (a) the initial $(\mathrm{M}=2.84, \mathrm{D}=5.76)$, (b) according to $a_{i}^{*}=1$ (1 iteration step. $\mathrm{M}=1.45$, $\mathrm{D}=0.34 ; 2$ iteration steps $\mathrm{M}=1.34, \mathrm{D}=0.06)$, (c) according to formula (4) (1 iteration step. $\mathrm{M}=1.78, \mathrm{D}=0.51 ; 2$ iteration steps. $\mathrm{M}=1.68, \mathrm{D}=0.37$ ).

\section{Examples and merits of the algorithm}

First, the usage of the projection operation together with quasi-statistical modeling allows preservation of all curvatures of the mesh geometry and main features of the given 3D object, while the mesh is smoothly improved without any additional criteria in the suggested algorithm for such surface preservation. It is a remarkable merit of the approach that is approved by our experimental results. Visual justification of one example can be found in Fig. 5. With the nearly same mean $\mathrm{M}=1.5$, the difference in volume between the initial model and the improved one is about $0.027 \%$ in case of using our technique and $0.37 \%$ after applying Laplacian smoothing. Moreover, in opposite to present method Laplacian algorithm continues smoothening the surface if more iterations are applied.

Next, as shown in Fig. 4, the implementation of the quasi-statistical modeling with another formula for the calculation can be used providing other results with 

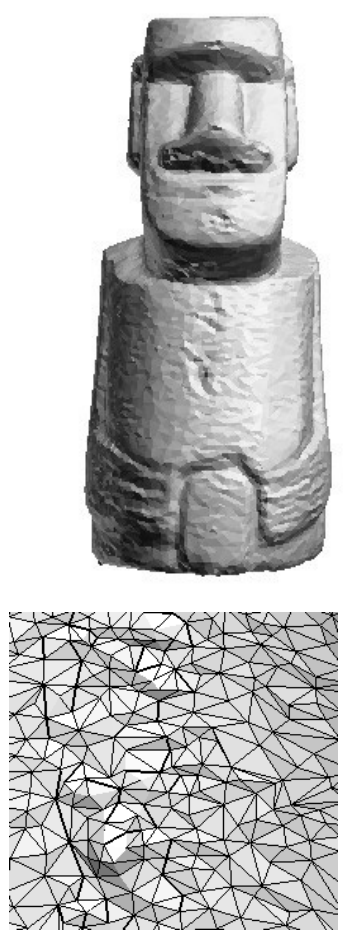

(a)
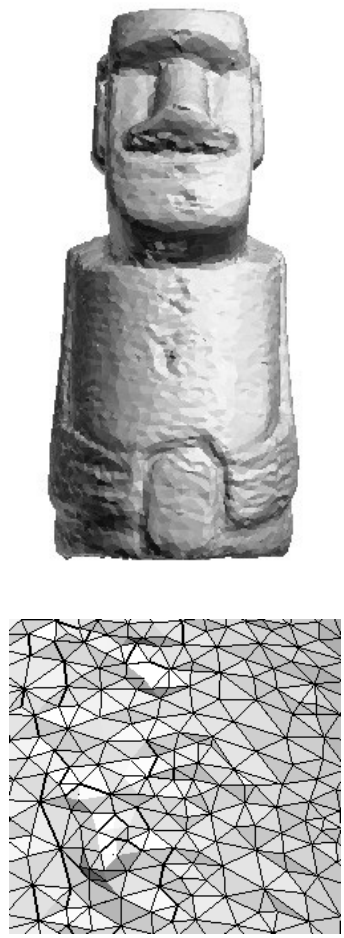

(b)
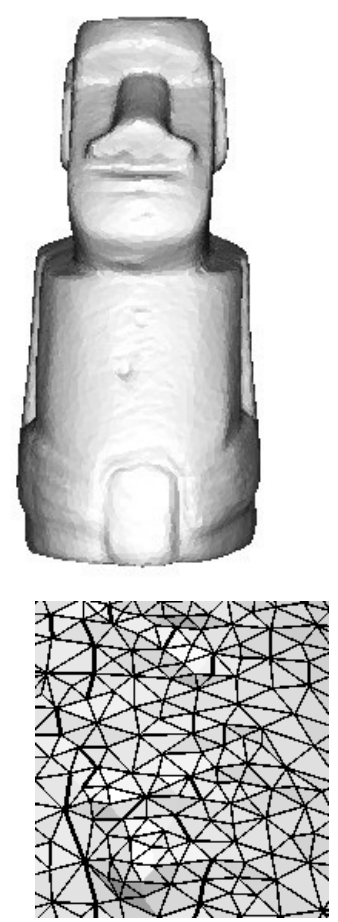

(c)

Fig. 5. Volume and surface preservation: (a) an intial model, (b) the proposed method, (c) Laplacian smoothing.

the same approach. For example, if $a_{i}^{*}=1$, it becomes similar to the Laplacian smoothing algorithm, which produces mostly equilateral elements by placing each point into the centroid of a star.

According to these two remarks, quasi-statistical modeling does not only provide smooth change from one element to another in the mesh improvement stage, but in addition it is a flexible independent tool for mesh processing. It can be combined with already existing methods not only in the mesh improvement but also, for example, in the mesh simplification case. We consider randomness as an invariant under the mesh improvement process. This approach offers the following opportunities: developing different mesh improvement algorithms based on quasistatistical modeling, considering meshes of any kind, selecting various mesh quality criteria parameters, realizing potential geometries with arbitrary choice of a star or by any special rule. For example, three variations of the present geometrical realization with potential forms are developed: Gentle Enhancement Algorithm (GEA), "Wave" and "Triangle-tail." Here we demonstrate only the GEA results to support the introduced explanation of the suggested concept. 
Experimental results of applying the GEA are demonstrated in Figs. 6-8. In the GEA, each star is selected according to the node's indices of the initial mesh generation. The described operation on a star is applied for each chosen star. Modeled values $a_{i}^{*}$ for each star are realized in the way explained ibid. Modeling random variable $\xi$ with Gaussian distribution is incarnated into the generated mesh. It is clear that the angles and the AR values have a correlation. Thus improving one parameter will cause corresponding distribution for other parameter. While most mesh elements have the AR values in the right-side neighbourhood of unity, the minimum angle values are normally distributed in the left-side neighbourhood of 60 degrees and maximum angle values - in the right-side of 60 degrees.
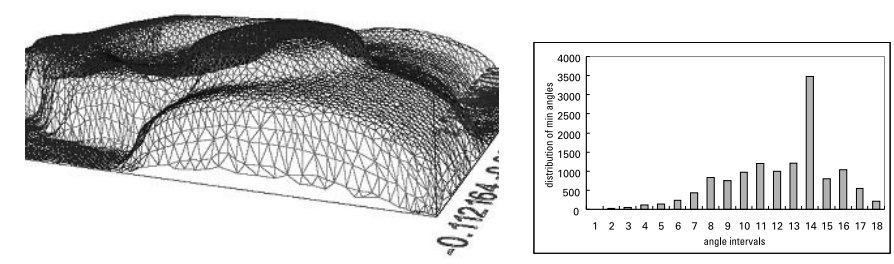

min angle

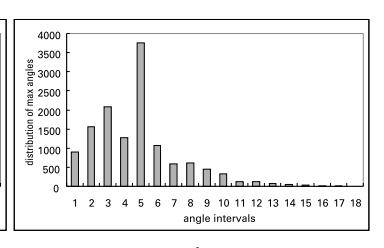

max angle

(a)

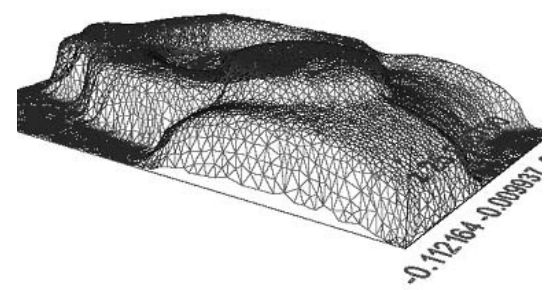

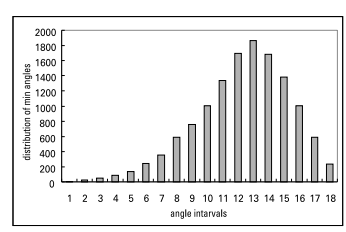

min angle

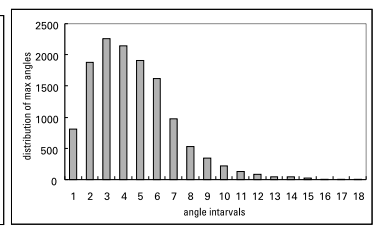

max angle

(b)

Fig. 6. Fragment of the mesh (the model "Detail," 13967 triangles) and the histograms of the distribution of the mesh element's angles $((x)$ axis - the number of the angle intervals; $(y)$ axis - the number of the mesh elements): (a) before use of GEA, $\mathrm{M}=1.56$. (b) after use of the GEA, $\mathrm{M}=1.53$. Processing time 4.0 sec. 1 iteration.

Table 1 shows statistics of the mesh angles for the model "Detail." All calculations are produced on Microsoft Windows XP, Pentium III, $1000 \mathrm{MHz} / 128 \mathrm{MB}$ RAM.

Table 1. Mesh angle statistic. The model "Detail."

\begin{tabular}{|c|c|c|c|c|}
\hline \multirow{2}{*}{} & \multicolumn{2}{|c|}{ Initial mesh } & \multicolumn{2}{c|}{ Using GEA } \\
\cline { 2 - 5 } & Average value & Angle range & Average value & Angle range \\
\hline Min angle (degree) & 40.69 & $5.25-59.62$ & 41.38 & $6.02-59.87$ \\
\hline Max angle (degree) & 86.44 & $60.65-166.75$ & 83.86 & $60.21-164.02$ \\
\hline
\end{tabular}



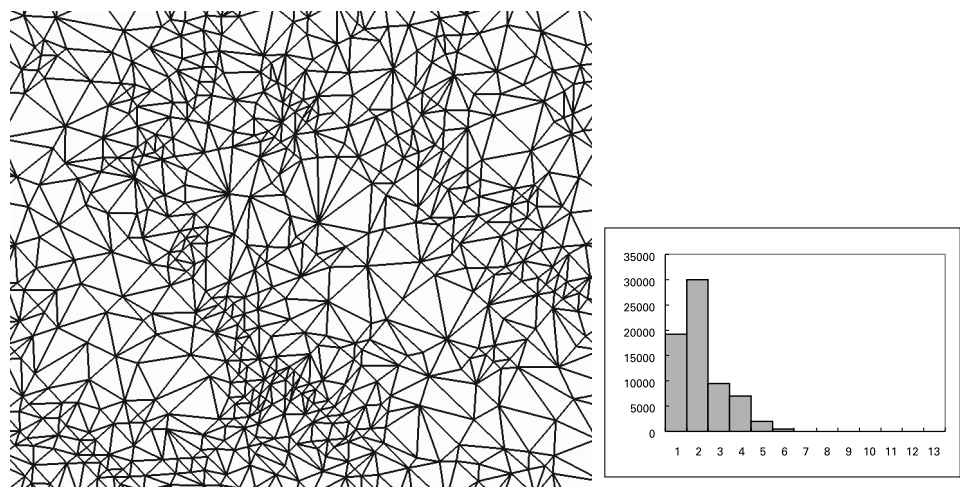

(a)
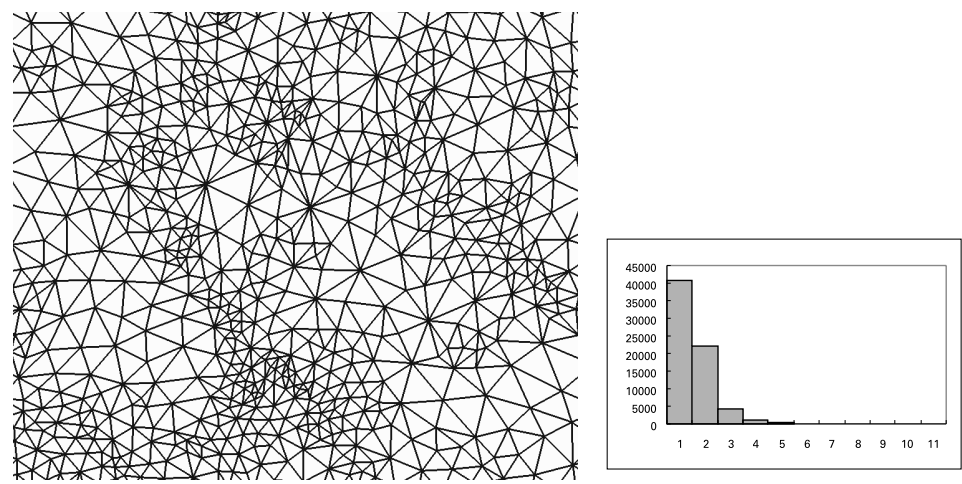

(b)

Fig. 7. Fragment of the mesh (the model "ballJoint," 68000 triangles) and the histogram of AR distribution $((x)$ axis - the number of the intervals of the AR value range $X=[1.0, \max ] ;(y)$ axis - the number of the mesh elements): (a) the initial mesh, $\mathrm{M}=1.84, \mathrm{D}=0.33$; (b) the same fragment after the use of the GEA, $\mathrm{M}=1.50$, $\mathrm{D}=0.16$. Processing time 30 sec. 1 iteration.

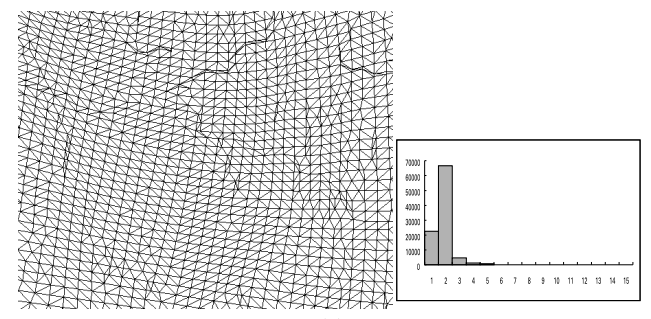

(a)

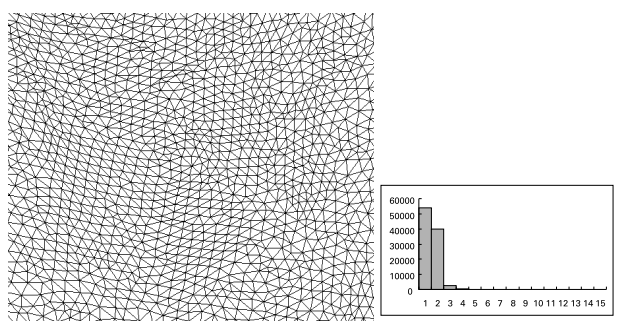

(b)

Fig. 8. Fragment of the mesh (model "horse," 96966 triangles) and the histogram of AR distribution $((x)$ axis - the number of the intervals of the AR value range $X=[1.0, \max ] ;(y)$ axis - the number of the mesh elements): (a) the initial mesh, $\mathrm{M}=1.73, \mathrm{D}=0.63$; (b) the same fragment after the use of the GEA, $\mathrm{M}=1.49, \mathrm{D}=0.36$. Processing time 50 sec. 1 iteration. 
In this paper we consider only triangle mesh improvement. Similar algorithm development is possible for other kinds of meshes (quadrilateral, mixed, solid). Some of the algorithms have been already realized.

Also from the result shown in Fig. 9, we can see that our technique can be applied for graded meshes and still produces a good mesh in terms of the mesh element control, reducing the number of low quality elements. In some complicated models, the worst value of the mesh quality parameter can deteriorate, but it only signifies the problematic areas of the mesh. The elements with high aspect ratio values are called the "tail" elements. They can be treated separately by topological improvement. Collapsing of such elements is done if allowed by requirements. Our algorithm works effectively in one or two iterations and it is developed for such direct application. For information, the effectiveness of the concept will be naturally decreased with the number of iterations.

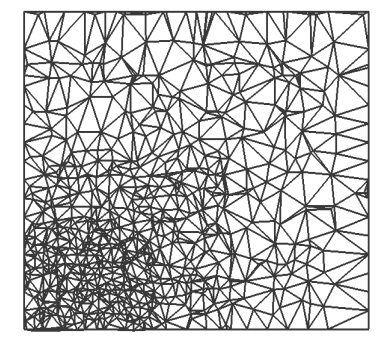

(a)

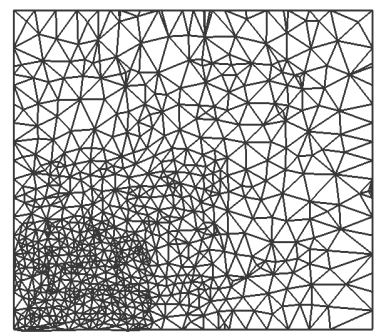

(c)

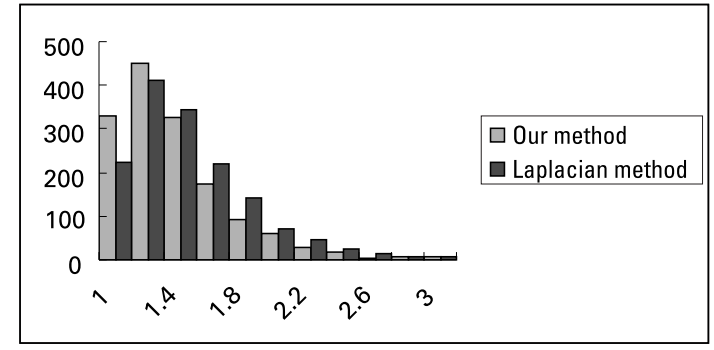

(b)

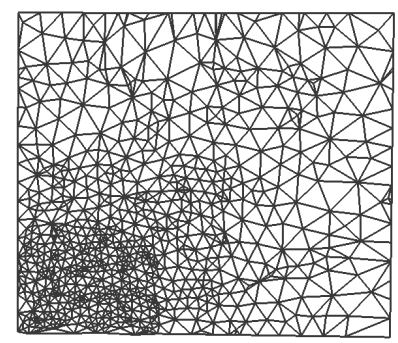

(d)

Fig. 9. The test model of smoothing graded mesh. (a) The initial mesh, $\mathrm{M}=2.46$; (b) AR histograms distribution $((x)$ axis - $\mathrm{AR}$ value, range $\mathrm{AR}=[1.0, \max ]$; (y) axis - the number of the mesh elements); (c) the mesh after Laplacian smoothing, 100 iterations, $\mathrm{M}=1.6$; (d) the mesh after using GEA, 2 iterations, $\mathrm{M}=1.5$.

\section{Concluding remarks}

In this paper, a new approach based on an implementation of quasi-statistical modeling for the triangular mesh improvement is investigated. Modeling of random variables with normal distribution based on random numbers and initial topology of the mesh is discussed. We select Gaussian function as a function appropriate for 
the distribution of all random numbers to form uniform meshes. The operation on a star is proposed for incarnation of modeling random variable. From a point of view of geometrical realization, our method can be used as a kind of the smoothing technique where the probabilistic direction of the point placement is calculated. One or two iteration steps of the algorithm are enough to attain the most effective results of the mesh improvement. This property can be also stated as a meaning advantage of the proposed method. Projection operation preserves the mesh geometry, volume and features of $3 \mathrm{D}$ objects. The suggested algorithm exhibits the satisfactory time performance. The computational complexity of the approach is almost linear in the number of points of the model according to the experiments.

Quasi-statistical modeling allows considering various mesh quality requirements and any kinds of meshes. Proposed method can be considered as an analogy with the simulation of the mesh improvement process through modeling of an event of mesh improvement. The further investigation may be devoted to the question of increasing possibility for such an event in opposite to experiments we have done till now. Many elliptic partial differential equations ideally require meshes with quasi-equilateral triangles (with the AR not so close to the ideal value). This kind of meshes is often desired in the field of simulation, for fluid flow or anisotropic diffusion for instance. It leaves subjects to be investigated depending on your demand for the mesh quality parameter.

Our technique can be used as a correction step for improving the mesh quality in the mesh simplification process. We applied this technique for the mesh simplification as a correction step for improving the mesh quality. In addition, this approach is currently being applied to isotropic meshes, but the application of it can be extended to anisotropic meshes (for instance, transient turbulent flow simulation) and to the meshes with various quality requirements.

Acknowledgements. The authors would like to thank A. Gonenc (Middle East Technical University, Turkey) for her useful recommendations concerning statistical applications. Special gratitude goes to E. Sedych (Lebedev Physics Institute of RAS, Russia) for fruitful discussions and comments.

\section{References}

[1] N. Amenta, M. Bern and D. Eppstein, Optimal point placement for mesh smoothing. J. Algorithms 30 (1999), 302-322.

[2] B. Balendran, A direct smoothing method for surface meshing. Proceedings of the 8th International Meshing Roundtable. South Lake Tahoe, CA, USA, 1999, 189-193.

[ 3 ] O. Egorova, M. Savchenko, N. Kojekine, I. Semenova, I. Hagiwaraand V. Savchenko, Improvement of mesh quality using a statistical approach. Proceedings of the 3th IASTED International conference on Vizualization. Imaging and Image Processing (VIIP), Spain, 2003, 1016-1021.

[4] P.J. Frey, About Surface Remeshing. Proceedings of the 9th International Meshing Roundtable. Sandia National Laboratories, 2000, 123-136.

[5] M. Holder and J. Richardson, Genetic algorithms, another tool for quad mesh optimization? Proceedings of the 7th International Meshing Roundtable. Sandia National Lab., 1998, 497-504. 
[ 6 ] F.J. Bossen and P.S. Heckbert, A Pliant method for anisotropic mesh generation. Proceedings of the 5th International Meshing Roundtable. Pittsburgh, PA, 1996, 63-74.

[ 7 ] D.A. Field, Laplacian smoothing and delaunay triangulations. J. Communications in Applied Numerical Methods, 4 (1998), 709-712.

[ 8 ] T. Zhou and K. Shimada, An angle-based approach to two-dimensional mesh smoothing. Proceedings of the 9th International Meshing Roundtable. 2000, 373-384.

[ 9 ] L.A. Freitag, On Combining Laplacian and Optimization-Based Mesh Smoothing Techniques. AMD 220, Trends in Unstructured Mesh Generation, 1997, 37-43.

[10] L.A. Freitag and C. Olliver-Gooch, A comparison of tetrahedral mesh improvement techniques. Proceedings of the 5th International Roundtable. Sandia National Lab., Albuquerque NM, 1996, 87-106.

[11] V. Parthasarathy and S. Kodiyalam, A constrained optimization approach to finite element mesh smoothing. J. Finite Elements in Analysis and Design, 9 (1991), 309-320.

[12] S.A. Canann, J.R. Tristano and M.L. Staten, An approach to combined Laplacian and optimization-based smoothing for triangular, quadrilateral, and quad-dominant meshes. Proceedings of the 7th International Meshing Roundtable, 1998, 479-494.

[13] O.P. Jacquotte and G. Coussement, Structured mesh adaptation: space accuracy and interpolation methods. Computer Methods in Applied Mechanics and Engineering, 101 (1992), 397-432.

[14] S.A. Canann, M.B. Stephenson and T.D. Blacker, Optismoothing: an optimization-driven approach to mesh smoothing. J. Finite Elements in Analysis and Design, 13 (1993), 185-190.

[15] R. Lohner, K. Morgan and O.C. Zienkiewicz, Adaptive Grid Refinement for the Euler and Compressible Navier Stokes Equation. Proceedings of the International Conference on Accuracy Estimates and Adaptive Refinement in Finite Element Computations. Lisbon, 1984.

[16] I. Babuska, O.C. Zienkiewicz, J. Gago and E.R. de A. Oliviera (eds.), Accuracy Estimates and Adaptive Refinements in Finite Element Computations. John Wiley \& Sons, Chichester, 1986, 281-297.

[17] K. Shimada and D.C. Gossard, Bubble mesh: automated triangular meshing of non-manifold geometry by sphere packing. Proceedings of the ACM Third Symposium on Solid Modeling and Applications. 1995, 409-419.

[18] K. Shimada, Anisotropic triangular meshing of parametric surfaces via close packing of ellipsoidal bubbles. Proceedings of the 6th International Meshing Roundtable. Sandia National Laboratories, 1997, 375-390.

[19] H.N. Djidjev, Force-directed methods for smoothing unstructured triangular and tetrahedral meshes. Proceedings of the 9th International Meshing Roundtable. Sandia National Laboratories, 2000, 395-406.

[20] G. Taubin, A signal processing approach to fair surface design. Proceedings of SIGGRAPH' 95, 29 (1995), 351-358.

[21] M. Desbrun, M. Meyer, P. Schrder and A.H. Barr, Implicit fairing of irregular meshes using diffusion and curvature flow. Proceedings of SIGGRAPH' 99, 33 (1999), 317-32.

[22] J. Warren and H. Weimer, Subdivision Methods for Geometric Design. Academic Press, 2002.

[23] L. Kobbelt, S. Campagna, J. Vorsatz and H-P. Seidel, Interactive multi-resolution modeling on arbitrary meshes. Proceedings of SIGGRAPH' 98, 32 (1998), 105-114.

[24] J.R. Shewchuk, What is a good linear element? interpolation, conditioning, and quality measures. Proceedings of the 11th International Meshing Roundtable. Sandia National Laboratories, 2002, 115-126.

[25] K.E. Jansen, M.S. Shepard, and M.W. Beall, On anisotropic mesh generation and quality control in complex flow problems. Proceedings of the 10th International Meshing Roundtable. Sandia National Laboratories, 2001, 341-349.

[26] I.M. Sobol, Monte Carlo Numerical Methods. Nauka, Moscow, 1973, in Russian.

[27] H. Hoppe, T. DeRose, T. Duchamp, J. McDonald and W. Stuetzle, Surface reconstruction from unorganized points. Proceedings of SIGGRAPH' 92, 1992, 71-78. 
